Rationalizability, Learning, and Equilibrium in Games with Strategic Complementarities Author(s): Paul Milgrom and John Roberts

Source: Econometrica, Vol. 58, No. 6 (Nov., 1990), pp. 1255-1277

Published by: The Econometric Society

Stable URL: http://www.jstor.org/stable/2938316

Accessed: 10/12/2010 19:03

Your use of the JSTOR archive indicates your acceptance of JSTOR's Terms and Conditions of Use, available at http://www.jstor.org/page/info/about/policies/terms.jsp. JSTOR's Terms and Conditions of Use provides, in part, that unless you have obtained prior permission, you may not download an entire issue of a journal or multiple copies of articles, and you may use content in the JSTOR archive only for your personal, non-commercial use.

Please contact the publisher regarding any further use of this work. Publisher contact information may be obtained at http://www.jstor.org/action/showPublisher?publisherCode=econosoc.

Each copy of any part of a JSTOR transmission must contain the same copyright notice that appears on the screen or printed page of such transmission.

JSTOR is a not-for-profit service that helps scholars, researchers, and students discover, use, and build upon a wide range of content in a trusted digital archive. We use information technology and tools to increase productivity and facilitate new forms of scholarship. For more information about JSTOR, please contact support@jstor.org. 


\title{
RATIONALIZABILITY, LEARNING, AND EQUILIBRIUM IN GAMES WITH STRATEGIC COMPLEMENTARITIES
}

\author{
By Paul Milgrom and John Roberts ${ }^{1}$
}

\begin{abstract}
We study a rich class of noncooperative games that includes models of oligopoly competition, macroeconomic coordination failures, arms races, bank runs, technology adoption and diffusion, R\&D competition, pretrial bargaining, coordination in teams, and many others. For all these games, the sets of pure strategy Nash equilibria, correlated equilibria, and rationalizable strategies have identical bounds. Also, for a class of models of dynamic adaptive choice behavior that encompasses both best-response dynamics and Bayesian learning, the players' choices lie eventually within the same bounds. These bounds are shown to vary monotonically with certain exogenous parameters.
\end{abstract}

KEYworDs: Game theory, supermodular games, iterated dominance, learning, strategic complements.

WE STUDY THE CLASS of (noncooperative) supermodular games introduced by Topkis (1979) and further analyzed by Vives $(1985,1989)$, who also pointed out the importance of these games in industrial economics. Supermodular games are games in which each player's strategy set is partially ordered, the marginal returns to increasing one's strategy rise with increases in the competitors' strategies (so that the game exhibits "strategic complementarity" ${ }^{2}$ ) and, if a player's strategies are multidimensional, the marginal returns to any one component of the player's strategy rise with increases in the other components. This class turns out to encompass many of the most important economic applications of noncooperative game theory.

In macroeconomics, Diamond's (1982) search model and Bryant's (1983, 1984) rational expectations models can be represented as supermodular games. In each of these models, more activity by some members of the economy raises the returns to increased levels of activity by others. In oligopoly theory, some models of Bertrand oligopoly with differentiated products qualify as supermodular games. In these games, when a firm's competitors raise their prices, the marginal profitability of the firm's own price increase rises. A similar structure is present in games of new technology adoption such as those of Dybvig and Spatt (1983), Farrell and Saloner (1986), and Katz and Shapiro (1986). When more users hook into a communication system or more manufacturers adopt an interface standard, the marginal return to others of doing the same often rises. Similarly, in some specifications of the bank runs model introduced by Diamond and Dybvig (1983), when more depositors withdraw their funds from a bank, it is more worthwhile for other depositors to do the same. In the warrant exercise

\footnotetext{
${ }^{1}$ We thank Michihiro Kandori, Andrew Postlewaite, Tim Bresnahan, Chris Shannon, and especially Don Brown for helpful conversations, the anonymous referees and an editor for helpful criticisms, and the NSF for financial support.

2 This term "strategic complements" was introduced by Bulow, Geanakoplos, and Klemperer (1985) to refer to games in which the best-response functions of the players are upward sloping. See also Fudenberg and Tirole (1986).
} 
model of Spatt and Sterbenz (1988), where the firm uses the proceeds of any exercised warrant to issue a special dividend to shareholders, the marginal return to exercising a warrant increases as other warrant holders choose to exercise. In the arm's race game modeled in Section 4, the marginal return to additional arms in any period is an increasing function of the foe's armament level in the same period and, moreover (given a convex cost of building new armaments), higher levels of armaments for one country in one period are complementary to higher levels for it in adjacent periods. ${ }^{3}$

Bayesian games provide another area of application. An example is Shavell's (1989) model of pretrial negotiations, in which the plaintiff decides whether to reveal its expected damages (its "type") before the trial begins and the defendant decides how much to offer as a settlement to silent plaintiffs. In that game, a strategy for the plaintiff is characterized by a single number-the highest level of expected damages for which the plaintiff remains silent-and a strategy for the defendant is the settlement offer to make to a silent plaintiff. It is more profitable for the plaintiff to adopt a "high" strategy when the settlement offered to silent plaintiffs is high, and it is more profitable for the defendant to make a high settlement offer when plaintiffs adopt a high strategy (because a higher offer is then more likely to deter a lawsuit). So, this too is a supermodular game.

The Shavell example is a Bayesian game in which supermodularity relies on the particular structure of the specification of uncertainty. Vives (1989) has shown that any Bayesian game derived from a parameterized family of supermodular games by adding uncertainty and private information about the payoff parameters is itself a supermodular game. As examples, the Diamond search model is still supermodular when there is uncertainty and private information about search costs and the arms race is still a supermodular game when there is uncertainty and private information about the cost or effectiveness of weapons.

The ordering of the strategy space is an important element in the formulation of supermodular games, just as it is in the formulation of supermodular optimization models (Granot and Veinott (1985), Milgrom and Roberts (1989)). For example, the multiperiod arms race game is supermodular when the strategic choices are ordered by the stock of arms held in each period (with the componentwise order), but not when it is ordered by the periodic level of investment in new armaments. As a second example, Vives (1989) has shown that Cournot duopoly games with a wide range of demand functions ${ }^{4}$ and arbitrary continuous cost functions are supermodular games if one of the two players' strategy sets is given the reverse of its usual order, but not with the standard order. As we show, a similar trick applies to the Hendricks-Kovenock

\footnotetext{
${ }^{3}$ Although we know of no game models in development economics, it would be reasonable to model Scitovsky's (1954) tale of the railroad and the steel companies, in which the value of an additional steel plant in a developing country depends (positively) on the investment in railroads and the value of additional investment in railroads increases with the level of investment in steel plants, as a supermodular game.

${ }^{4}$ Specifically, these are the demand functions for which an increase in one player's quantity reduces the other's marginal revenue.
} 
(1989) oil exploration externality game-a Bayesian game in which the increased likelihood of early exploratory drilling activity by one firm makes it more profitable for the other to postpone drilling to await the first firm's results before deciding on its own drilling program. Only if the strategies are ordered so that more frequent drilling is a "higher" strategy for one player and less frequent drilling is a higher strategy for the other is the game supermodular.

Additional applications of the theory are possible once we recognize that, although supermodularity itself is a cardinal concept, our analysis of supermodular games is entirely ordinal in character, that is, it uses only inequalities among payoffs to pure strategy profiles. For example, Reinganum's (1981) R \& D competition game, in which firms race to be the first to patent an innovation, is not a supermodular game, but the corresponding game in which the payoffs are replaced by their logarithms is supermodular. Consequently, all of our conclusions about supermodular games will apply to Reinganum's game. In Section 4, we use a similar trick to show Bertrand oligopoly games are log-supermodular if each firm's elasticity of demand is a decreasing function of its competitors' prices. The class of demand functions satisfying this restriction includes linear, logit, CES, and translog demand specifications, and others. Additional examples of supermodular games are reported by Cooper and John (1988), Lippman, Mamer, and McCardle (1987), Sobel (1989), Topkis (1979), and Vives (1989).

The centerpiece of our analysis is Theorem 5. It establishes that the set of serially undominated strategy profiles, that is, those that remain after an iterative procedure of crossing out strongly dominated strategies, has a maximum and minimum element, and that these elements are Nash equilibria. It is well known that all the major approaches to noncooperative equilibrium predict outcomes in the serially undominated set; the set includes all the pure and mixed strategy Nash equilibria, all the correlated equilibria (both subjective and objective; see Aumann (1987)), and all the rationalizable strategies (Bernheim (1985), Pearce (1985)). Since pure strategy Nash equilibria are also solutions under these various concepts, it follows that all of these approaches predict the same upper and lower bounds on the joint behavior of players in supermodular games. If the game is symmetric, then the bounds on the set of serially undominated strategies are symmetric pure strategy Nash equilibria, so the bounds on joint behavior predicted by this symmetric equilibrium concept coincide with those predicted by all the other (possibly asymmetric) solution concepts.

An alternative approach to solution concepts is to treat the set of strategies that are played infinitely often in some dynamic adjustment process as a solution. For a very wide class of such processes-a class we call adaptive dynamics and that includes best-response dynamics, fictitious play, Bayesian learning, and many others-we show that the bounds on the solution set in supermodular games are always the same Nash equilibria that bound the serially undominated set. Then, the predicted bounds on eventual behavior under adaptive dynamic theories coincide with the predicted bounds of all the other noncooperative solution concepts. 
The usefulness of Theorem 5 depends partly on how wide the bounds are that we obtain. Indeed, for some games, these bounds are so wide that our result is of little help: it is even possible that the maximum and minimum elements of the strategy space are equilibria. In that case, none of the theories impose any tight bounds on joint behavior. However, for other games, the bounds are quite narrow. For example, in the applications section, we show that there can be only one symmetric pure strategy Nash equilibrium in the arms race game described in Section 4 and only one pure strategy Nash equilibrium in the (possibly asymmetric) Bertrand pricing games with linear, logit, or CES demand. It then follows that these games possess no other equilibria (whether symmetric or asymmetric, mixed or pure, Nash or correlated), that each player has only one rationalizable strategy, and that any adaptive dynamic process leads to behavior that converges from any starting point to the unique equilibrium.

In economic modelling, the analyst is usually interested not only in the existence, range, and stability of equilibria and the comparisons among different solution concepts covered by the cited theorems, but also in comparative statics and in the welfare properties of the equilibria. We offer two theorems to aid in analyses of this sort. The first (Theorem 6) provides a useful general condition under which the bounds of the equilibrium set vary monotonically with an exogenous parameter. The second (Theorem 7) is a welfare theorem. One of its implications is that there is a Pareto-best and a Pareto-worst equilibrium if each player's payoff is a nondecreasing function of the others' strategy choices. For example, in the Bertrand pricing game, all firms prefer the equilibrium with the highest prices and in the macroeconomic games, all parties prefer the equilibrium with the highest level of economic activity. Another implication is that if some players' payoffs are increasing and some are decreasing in the others' strategies, then there is a fundamental conflict among the players: the equilibrium with the highest payoff for one group is the equilibrium with the lowest payoff for the other. For example, in the Cournot duopoly game the equilibrium with the highest payoff for one firm is the one in which its output is highest and its competitor's output (and payoff) is lowest. A similar result obtains in the Hendricks-Kovenock drilling game. In that game, a firm that drills an early exploratory well supplies free information to the other firm, so the equilibrium with the highest payoff for firm 2 and the lowest payoff for firm 1 is the equilibrium in which firm 1 drills early most often and firm 2 drills early least often. Also, in the technology adoption game, the equilibrium with the most extensive adoptions of the new technology is the equilibrium most preferred by players who were ill-served by the older technology and least preferred by players who were well served by it.

Our analysis of supermodular games also unearths a subsidiary result of independent mathematical interest. With Theorems 1 and 2, we establish that a bounded, order upper semi-continuous, supermodular function on a complete lattice has a maximum and that the set of maximizers is a complete sublattice. These theorems are essential ingredients in the proof of Theorem 5. 
A number of other papers have developed results related to ours. Topkis (1979) showed that continuous, supermodular games with finite-dimensional, compact intervals for strategy spaces have increasing best-response functions ${ }^{5}$ and used this result to establish the existence of pure strategy Nash equilibrium points for these games. He showed that the set of such equilibrium points has largest and smallest elements and also provided a computational algorithm for finding these. Lippman, Mamer, and McCardle (1987) and Sobel (1989) have established comparative statics theorems, showing the existence of a monotone selection from the equilibrium set as certain parameters are varied. ${ }^{6}$ Cooper and John (1988) proved a version of the welfare theorem for supermodular games in an analysis of models of macroeconomic coordination failures. Moulin (1984) and Bernheim (1984) studied the relationship between dominance solvability and best-response stability of equilibrium in a class of smooth games including Cournot games. Vives (1989) employs results of Hirsch $(1982,1985)$ to show that a kind of continuous adjustment process converges to an equilibrium for "strictly" supermodular games played on convex domains. Independently of our work, Gul (1988) has shown for general finite games that there is a large class of adaptive learning models for which the long-run behavior of the players lies entirely in the class of rationalizable strategies. Besides these theoretical contributions, many of the applications papers developed their particular results using arguments that have proven to be generalizable. Our analysis adds to these predecessors by weakening slightly the definition of supermodular games, contributing many new examples, encompassing log-supermodular and other ordinally transformed supermodular games within the theory, extending the analysis from pure Nash equilibria to include mixed, correlated, and rationalizable strategies, and introducing a comprehensive theory of adaptive dynamics applicable to this class of games.

The remainder of this paper is organized as follows. Section 1 presents the mathematical preliminaries. It begins with the basic definitions of lattice theory and then reports some previously known results in the theory and our new results. Section 2 presents the basic theory of supermodular games, including the main theorem on the existence of equilibrium and the equivalence of equilibrium concepts and the theorems on comparative statics and welfare. Section 3 introduces the model of adaptive behavior for games in general and identifies its implications for supermodular games in particular. Section 4 provides detailed analyses of five examples of supermodular games that apply and develop our various results. Concluding remarks are presented in Section 5.

\footnotetext{
${ }^{5}$ More precisely, he showed that the best response correspondence has values which are nonempty compact sublattices, so that there is always a largest and smallest best response. In addition, he showed that these largest and smallest best response functions are nondecreasing.

${ }^{6}$ Lippman, Mamer, and McCardle treat the case of games with monotone best response functions while Sobel treats the more general case of monotone best response correspondences, appropriately defined.
} 
Readers primarily interested in applications using the Nash equilibrium concept may skip the mathematical preliminaries and begin with Section 2, which includes a characterization of what might be termed the class of "smooth supermodular games" (including most of the applications that we have identified) and also presents the main theorem. They may then continue with Section 3 , which analyzes adaptive behavior, and Section 4, which illustrates various applications.

\section{MATHEMATICAL PRELIMINARIES}

Consider a set $S$ with a partial order $\geqslant$ that is transitive, reflexive, and antisymmetric. ${ }^{7}$ Given $T \subset S, \bar{b} \in S$ is called an upper bound for $T$ if $\bar{b} \geqslant x$ for all $x \in T$; it is the supremum of $T$ (denoted $\sup (T)$ ) if for all upper bounds $b$ of $T, b \geqslant \bar{b}$. Lower bounds and infimums are defined analogously. A point $x$ is a maximal element of $S$ if there is no $y \in S$ such that $y>x$ (that is, no $y$ such that $y \geqslant x$ but not $x \geqslant y$ ); it is the largest element of $S$ if $x \geqslant y$ for all $y \in S$. Minimal and smallest elements are defined similarly. A set may have many maximal and minimal elements, but it can have at most one largest element and one smallest element.

The set $S$ is a lattice if for each two point set $\{x, y\} \subset S$, there is a supremum for $\{x, y\}$ (denoted $x \vee y$ and called the join of $x$ and $y$ ) and an infimum (denoted $x \wedge y$ and called the meet of $x$ and $y$ ) in $S$. The lattice is complete if for all nonempty subsets $T \subset S, \inf (T) \in S$ and $\sup (T) \in S$. An interval is a set of the form $[x, y] \equiv\{z \mid y \geqslant z \geqslant x\}$.

The real line (with the usual order) is a lattice and any compact subset of it is, in fact, a complete lattice, as is any set in $\Re^{n}$ formed as the product of $n$ compact sets (with the product order). The interval $(0,1)$ is a lattice which is not complete, while $\left\{\left(x_{1}, x_{2}\right) \in \mathfrak{R}^{2} \mid x_{1}+x_{2} \leqslant 1\right\}$ is a simple example of a set which is not a lattice.

A sublattice $T$ of a lattice $S$ is a subset of $S$ that is closed under $\wedge$ and $\vee$. A complete sublattice $T$ is a sublattice such that the infimum and supremum of every subset of $T$ is in $T$. A subset that is a lattice or complete lattice in its own right may not be a sublattice or complete sublattice of a larger lattice, because the relevant sup's and inf's are defined relative to the original, larger lattice. Thus, the set $T=[0,1) \cup\{2\}$ is a complete lattice under the usual ordering; the least upper bound in $T$ for the set $[0,1)$ is $2 \in T$. However, $T$ is not a complete sublattice of $[0,2]$ because then $\sup [0,1)=1 \notin T$. Similarly, under the usual ordering on $\Re^{2}$, the set $T=\{(0,0),(1,0),(0,1),(2,2)\}$ is a lattice but not a sublattice of $\Re^{2}$ because $(1,0) \vee(0,1)=(1,1) \notin T$.

A chain $C \subset S$ is a totally ordered subset of $S$, that is, for any $x \in C$ and $y \in C, x \geqslant y$ or $y \geqslant x$. Given a complete lattice $S$, a function $f: S \rightarrow \mathfrak{R}$ is order continuous if it converges along every chain $C$ (in both the increasing and

\footnotetext{
${ }^{7}$ Recall that transitive means that $x \geqslant y$ and $y \geqslant z$ imply $x \geqslant z$; reflexive means that $x \geqslant x$; and antisymmetric means that $x \geqslant y$ and $y \geqslant x$ implies $x=y$.
} 
decreasing directions), ${ }^{8}$ that is, if $\lim _{x \in C, x \downarrow \inf (C)} f(x)=f(\inf (C))$ and $\lim _{x \in C, x \uparrow \sup (C)} f(x)=f(\sup (C))$. It is order upper semi-continuous if $\lim \sup _{x \in C, x \downarrow \inf (C)} f(x) \leqslant f(\inf (C))$ and $\lim \sup _{x \in C, x \uparrow \sup (C)} f(x) \leqslant f(\sup (C))$.

A function $f: S \rightarrow \Re$ is supermodular if for all $x, y \in S$,

$$
f(x)+f(y) \leqslant f(x \wedge y)+f(x \vee y) .
$$

Supermodularity represents the economic notion of complementary inputs. The theory of supermodular optimization has been developed by Topkis (1978) and Granot and Veinott (1985). The following result characterizes supermodularity in differential terms for the case of smooth functions with Euclidean domains. The standard order on such domains is the "product order" according to which $x \geqslant y$ iff $x_{i} \geqslant y_{i}$ for all $i$.

Topkis's Characterization Theorem: Let $I=[\underline{x}, \bar{x}]$ be an interval in $\mathfrak{R}^{n}$. Suppose that $f: \Re^{n} \rightarrow \mathfrak{R}$ is twice continuously differentiable on some open set containing $I$. Then $f$ is supermodular on $I$ if and only if for all $x \in I$ and all $i \neq j$, $\partial^{2} f / \partial x_{i} \partial x_{j} \geqslant 0$. (There are no restrictions on $\partial^{2} f /\left(\partial x_{i}\right)^{2}$.)

In general, supermodularity, like all the concepts in this theory, uses only the order structure of the lattice. It entails no assumptions of convexity or even connectedness of the domain, nor does it require any convexity, concavity, or differentiability of the function itself. However, in view of the theorem, it is particularly easy to check whether smooth functions on Euclidean intervals are supermodular. ${ }^{9}$

Given two lattices $S_{1}$ and $S_{2}$, a function $f: S_{1} \times S_{2} \rightarrow \Re$ has increasing differences in its two arguments $x$ and $y$ if for all $x \geqslant x^{\prime}$, the difference $f(x, y)-f\left(x^{\prime}, y\right)$ is nondecreasing in $y .{ }^{10}$ In the game model that follows, if $x$ is interpreted as one player's strategy, $y$ as the other's, and $f$ as the first player's payoff, then the assumption of increasing differences is essentially the assumption of strategic complementarity: When the second player increases his choice variable(s), it becomes more profitable for the first to increase his as well.

Some basic results for functions on lattices that we shall need are as follows.

TARski's Fixed Point Theorem: If $T$ is a complete lattice and $f: T \rightarrow T$ is a nondecreasing function, then $f$ has a fixed point. Moreover, the set of fixed points

\footnotetext{
${ }^{8}$ The definition of order continuity is traditionally given in the stricter form that $f$ must converge along all convergent nets, rather than just along chains (cf. Birkhoff (1967)). Our "weaker" requirement is actually equivalent to convergence along nets when the domain of the function is a complete lattice. Similarly, it can be shown that a lattice is complete if every chain (rather than every nonempty set) has a supremum and an infimum in the lattice.

${ }^{9}$ In Milgrom and Roberts (1989), we have weakened the hypotheses of Topkis's Characterization Theorem as follows. The assumption that the domain of $f$ is an interval is replaced by the assumption that it is a path-connected sublattice of $\mathfrak{R}^{n}$ and the assumption that $f$ is twice continuously differentiable is replaced by the assumption that, for each two arguments $x_{i}$ and $x_{i}, f$ can be expressed as the indefinite integral of over these arguments of some nonnegative function $f_{i j}$. These small extensions have proved to be important for certain applications.

${ }^{10}$ Note that this is equivalent to the condition that $f(x, y)-f\left(x, y^{\prime}\right)$ is nondecreasing in $x$ for all $y \geqslant y^{\prime}$.
} 
of $f$ has $\sup \{x \in T \mid f(x) \geqslant x\}$ as its largest element and $\inf \{x \in T \mid f(x) \leqslant x\}$ as its smallest element.

Topkis's Monotonicity TheOREM: Let $S_{1}$ be a lattice and $S_{2}$ a partially ordered set. Suppose $f(x, y): S_{1} \times S_{2} \rightarrow \Re$ is supermodular in $x$ for given y and has increasing differences in $x$ and $y$. Suppose that $y \geqslant y^{\prime}$ and that $x \in M \equiv$ $\operatorname{argmax} f(x, y)$ and $x^{\prime} \in M^{\prime} \equiv \operatorname{argmax} f\left(x, y^{\prime}\right)$. Then $x \wedge x^{\prime} \in M^{\prime}$ and $x \vee x^{\prime} \in M$. In particular (when $y=y^{\prime}$ ), the set of maximizers of $f$ is a sublattice.

Some new results in the theory of optimization on lattices will be needed in the analysis below:

THEOREM 1: If $f$ is an order upper semi-continuous, supermodular function from a complete lattice $S$ to $\mathfrak{R} \cup\{-\infty\}$, then $f$ has a maximum on $S$.

Proof: Shannon (1989) has shown that the hypotheses of Theorem 1 imply that $f$ has a finite least upper bound, which we may take to be zero. We show here that there exists $\bar{y} \in S$ such that $f(\bar{y})=0$.

Let $\left\{x_{n}\right\}$ be a sequence with the property that $f\left(x_{n}\right) \geqslant-2^{-n}$. Define

$$
y_{n m}=x_{n} \wedge \cdots \wedge x_{n+m} .
$$

Since $y_{n m}$ is decreasing in $m$ and $S$ is complete, the sequence has a limit $y_{n} \in S$. By order upper semi-continuity, $\lim \sup _{m \rightarrow \infty} f\left(y_{n m}\right) \leqslant f\left(y_{n}\right)$.

We claim that $f\left(y_{n m}\right) \geqslant-\left(2^{-n}+\cdots+2^{-(n+m)}\right)$. Indeed, it is evident that $f\left(y_{n 0}\right)=f\left(x_{n}\right) \geqslant-2^{-n}$, and we proceed inductively:

$$
\begin{aligned}
f\left(y_{n m}\right) & =f\left(y_{n, m-1} \wedge x_{n+m}\right) \\
& \geqslant f\left(y_{n, m-1}\right)+f\left(x_{n+m}\right)-f\left(y_{n, m-1} \vee x_{n+m}\right) \\
& \geqslant-\left(2^{-n}+\cdots+2^{-(n+m-1)}\right)-2^{-(n+m)}-0,
\end{aligned}
$$

where we have used the definition of $y_{n m}$, supermodularity, and the fact that $f$ is bounded above by zero. By order upper semi-continuity, $f\left(y_{n}\right) \geqslant$ $\lim \sup _{m} f\left(y_{n m}\right) \geqslant-2^{1-n}$.

Observe that $y_{n}=\inf _{m \geqslant n} x_{m}$, so it is increasing in $n$. Hence, because $S$ is complete, $\left\{y_{n}\right\}$ has a sup in $S$, namely $\bar{y}=\lim y_{n}$, and $0=\sup f \geqslant f(\bar{y}) \geqslant$ $\lim \sup f\left(y_{n}\right) \geqslant 0$, so $\bar{y}$ attains the maximum.

Q.E.D.

The following example, suggested by Michihiro Kandori, shows that it is not in general true that a bounded, order upper semi-continuous function from a complete lattice to $\Re$ has a maximum. Let $S=\{(0,0),(1,1)\} \cup\left\{x \mid x_{1}+x_{2}=\right.$ $\left.1, x_{1}, x_{2}>0\right\}$. Using the component-wise ordering, it is easy to check that $S$ is a complete lattice: For any set $T$ containing two points on the diagonal portion, $\sup (T)=(1,1)$ and $\inf (T)=(0,0)$. Order-continuity is no help: no chain on $S$ 
has more than three elements, so every function $f: S \rightarrow \mathfrak{R}$ is order-continuous. Nevertheless, if $f$ is bounded and supermodular, then it has a maximum. ${ }^{11}$

THEOREM 2: If $f$ is an order upper semi-continuous, supermodular function from a complete lattice $S$ to $\mathfrak{R} \cup\{-\infty\}$, then the set of maximizers of $f$ is a complete sublattice of $S$.

Proof: Let $T$ be the set of maximizers of $f$, which by Theorem 1 is nonempty. It follows from Topkis's Monotonicity Theorem that $T$ is a sublattice of $S$.

Let $V$ be an arbitrary subset of $T$. It follows by order-continuity that every chain $C \subset \hat{T}=T \cap\{x \mid x \leqslant \sup (V)\}$ has $\sup (C) \in \hat{T}$. Hence, by Zorn's Lemma, ${ }^{12}$ $\hat{T}$ has a maximal element $\hat{x}$. Since $\hat{T}$ is a sublattice, this $\hat{x}$ must actually be the largest element in $\hat{T}$ (for if $y \in \hat{T}$ and $\hat{x} \neq y$, then $\hat{x} \vee y \in \hat{T}$ and $\hat{x} \vee y>\hat{x}$, contradicting the maximality of $\hat{x})$. By construction, $\sup (\hat{T})=\sup (V) \in \hat{T} \subset T$. Similarly, $\inf (V) \in T$ and hence $T$ is complete.

Q.E.D.

Corollary: Let $S$ be a complete lattice and suppose $f: S \rightarrow \Re \cup\{-\infty\}$ is order upper semi-continuous and supermodular. Then there are largest and smallest elements of the set of maximizers of $f$.

An especially useful application of these results arises in $L_{p}$-space. Let $L_{p}\left([0,1], \Re^{k}\right)$ be the set of functions $f:[0,1] \rightarrow \Re^{k}$ such that $\|f\|=$ $\left(f|f(x)|^{p} d x\right)^{1 / p}<\infty$ where $|f(x)|$ refers to the Euclidean norm. We identify any two functions $f$ and $g$ such that $\|f-g\|=0$. Let us say that $f \geqslant g$ if $\{x \mid g(x)>$ $f(x)\}$ is a null set. Then the following results are well known (cf. Aliprantis and Burkinshaw (1985)):

THEOREM 3: Suppose $1 \leqslant p<\infty$. Then, the $L_{p}$ norm is order-continuous. Moreover, any interval $[x, y]$ in $L_{p}$ is a complete lattice.

Corollary: Suppose that $f: L_{p} \rightarrow \Re \cup\{-\infty\}$ is an $L_{p}$-upper semi-continuous, supermodular function on an interval $[x, y]$. Then $f$ has a maximum and the set of maximizers is a nonempty, complete sublattice of $[x, y]$.

Proof: Apply Theorems 1-3.

Q.E.D.

\section{SOLUTIONS OF SUPERMODULAR GAMES}

The objects of our study are games in what we shall call "ordered normal form." These are described as follows. Let $N \neq \varnothing$ be the set of players; $N$ may

\footnotetext{
${ }^{11}$ For example, the linear function $g\left(x_{1}, x_{2}\right)=x_{1}-x_{2}$ is not supermodular with the specified order, and it has no maximum on $S$. The linear function $g\left(x_{1}, x_{2}\right)=x_{1}+x_{2}$ is supermodular, and it does have a maximum on $S$.

${ }^{12}$ Zorn's Lemma is as follows: If $S$ is a partially ordered set with the property that every chain in $S$ has an upper bound, then $S$ has a maximal element.
} 
be finite or infinite. Each player $n \in N$ has a strategy set $S_{n}$ with typical element $x_{n}$; the competitors' strategies are denoted by $x_{-n}$ and a full strategy profile is denoted by $x=\left(x_{n}, x_{-n}\right) \in S$. Elements of $S$ are called strategy profiles. Each strategy set $S_{n}$ comes with a partial order $\geqslant_{n}$, which we will later denote more simply by $\geqslant$, and the strategy profiles are endowed with the product order, that is, $x \geqslant x^{\prime}$ means $x_{n} \geqslant{ }_{n} x_{n}^{\prime}$ for all $n \in N$. Player $n$ 's payoff function is $f_{n}\left(x_{n}, x_{-n}\right)$. The object $\Gamma=\left\{N,\left(S_{n}, f_{n}, n \in N\right), \geqslant\right\}$ is a game in ordered normal form. The game $\Gamma$ is a supermodular game if, for each $n \in N$ :

(A1) $S_{n}$ is a complete lattice;

(A2) $f_{n}: S \rightarrow \Re \cup\{-\infty\}$ is order upper semi-continuous in $x_{n}$ (for fixed $x_{-n}$ ) and order continuous in $x_{-n}$ (for fixed $x_{n}$ ) and has a finite upper bound;

(A3) $f_{n}$ is supermodular in $x_{n}$ (for fixed $x_{-n}$ );

(A4) $f_{n}$ has increasing differences in $x_{n}$ and $x_{-n}$.

For many games of interest, the conditions of supermodularity can be easily checked using Theorem 4 below. Thus, suppose that a typical strategy for player $n$ is $\left(x_{n j} ; j=1, \ldots, k_{n}\right) \in \mathfrak{R}^{k_{n}}$ and that $\geqslant$ is the usual componentwise ordering.

THEOREM 4: Suppose there are finitely many players and the strategies and orders are as described in the preceding paragraph. Then $\Gamma$ is supermodular if $\left(\mathrm{A} 1^{\prime}\right)-\left(\mathrm{A} 4^{\prime}\right)$ below are satisfied:

$\left(\mathrm{A} 1^{\prime}\right) S_{n}$ is an interval in $\Re^{k_{n}}$, that is,

$$
S_{n}=\left[\underline{y}_{n}, \bar{y}_{n}\right]=\left\{x \mid \underline{y}_{n} \leqslant x \leqslant \bar{y}_{n}\right\}
$$

(A2') $f_{n}$ is twice continuously differentiable on $S_{n}$;

(A3') $\partial^{2} f_{n} / \partial x_{n i} \partial x_{n j} \geqslant 0$ for all $n$ and all $1 \leqslant i<j \leqslant k_{n}$;

(A4') $\partial^{2} f_{n} / \partial x_{n i} \partial x_{m j} \geqslant 0$ for all $n \neq m, 1 \leqslant i \leqslant k_{n}$ and $1 \leqslant j \leqslant k_{m}$.

Proof: Immediate from Topkis's Characterization Theorem.

Q.E.D.

Games satisfying (A1')-(A4') will be called smooth supermodular games.

A pure Nash equilibrium is a strategy tuple $x=\left(x_{n} ; n \in N\right)$ such that each $x_{n}$ maximizes $f\left(\hat{x}_{n}, x_{-n}\right)$ over $S_{n}$. Any pure Nash equilibrium is (by definition) also a mixed Nash equilibrium and a correlated equilibrium, although there may exist mixed equilibria that are not pure and correlated equilibria that are not mixed. The sets of strategies $\bar{S}_{m} \subset S_{m}, m=1, \ldots, N$, are rationalizable if for each $n$ and $x_{n} \in \bar{S}_{n}, x_{n}$ maximizes $E\left[f_{n}\left(\cdot, x_{-n}\right)\right]$ for some probability distribution on $x_{-n}$ with support in $\bar{S}_{-n}$. A strategy is rationalizable if it belongs to some rationalizable set. The component strategies of a pure Nash equilibrium are all rationalizable; the rationalizable sets of strategies consist of the singletons defined by the equilibrium point. The pure Nash equilibria are included as possible solutions under any of the solution concepts mentioned above.

A pure strategy $x_{n}$ for player $n$ is said to be strongly dominated by another pure strategy $\hat{x}_{n}$ if it is the case that for all $x_{-n}, f\left(x_{n}, x_{-n}\right)<f\left(\hat{x}_{n}, x_{-n}\right)$. A 
rational player would always prefer playing the dominating strategy $\hat{x}_{n}$ to the dominated strategy $x_{n}$. Given a product set $\hat{S}$ of strategy profiles, we define the set of $n$ 's undominated responses to $\hat{S}$ by $U_{n}(\hat{S})=\left\{x_{n} \in S_{n} \mid\left(\forall x_{n}^{\prime} \in S_{n}\right)\right.$ $\left.(\exists \hat{x} \in \hat{S}) f_{n}\left(x_{n}, \hat{x}_{-n}\right) \geqslant f_{n}\left(x_{n}^{\prime}, \hat{x}_{-n}\right)\right\}$. Let $U(\hat{S})=\left(U_{n}(\hat{S}) ; n \in N\right)$ be the list of undominated responses for each player, and let $\bar{U}(\hat{S})$ denote the interval $[\inf (U(\hat{S})), \sup (U(\hat{S}))]$.

We may use $U$ to represented the process of iterated elimination of strongly dominated strategies, as follows. Define $S^{0}=S$, the full set of strategy profiles. For $\tau \geqslant 1$, define $S^{\tau}=U\left(S^{\tau-1}\right)$. A strategy $x_{n}$ is serially undominated if $x_{n} \in$ $U_{n}\left(S^{\tau}\right)$ for all $\tau$; these are just the strategies that survive the iterative process of crossing out strongly dominated strategies. Observe that $U$ is a monotone nondecreasing function, that is, if $\hat{S} \subset S^{\prime}$, then $U(\hat{S}) \subset U\left(S^{\prime}\right)$. It is well known that only serially undominated strategies can be rationalizable and only serially undominated strategies can be played with positive probability at a pure Nash equilibrium, mixed Nash equilibrium, or correlated equilibrium.

In most treatments, a strategy $x_{n}$ for player $n$ is considered to be strongly dominated if it is dominated by a pure or mixed strategy, that is, if there exists a probability distribution over pure strategies for $n$ such that for all $x_{-n}, E\left[f_{n}\left(\tilde{x}_{n}, x_{-n}\right)\right]>f_{n}\left(x_{n}, x_{-n}\right)$, where the expectation is taken with respect to the probability distribution. The dominance solution of the game is the set of strategies remaining after iterative application of this process and the game is called dominance solvable if the dominance solution consists of a single point. The dominance solution, like all the others we have considered, contains the Nash equilibrium strategies and is contained in the set of serially undominated strategies.

The following Theorem is the key to the rest of our analysis.

THEOREM 5: Let $\Gamma$ be a supermodular game. For each player $n$, there exist largest and smallest serially undominated strategies, $\bar{x}_{n}$ and $\underline{x}_{n}$. Moreover, the strategy profiles $\left(\underline{x}_{n} ; n \in N\right)$ and $\left(\bar{x}_{n} ; n \in N\right)$ are pure Nash equilibrium profiles.

According to the Theorem, all serially undominated strategies (and thus all rationalizable and equilibrium strategies) lie in an interval $[\underline{x}, \bar{x}]$ whose maximum and minimum points are the largest and smallest Nash equilibria. We state the first step of the proof as a lemma, since it will prove to be useful for later developments. (Recall that $[x, y]$ designates the interval of points $\geqslant x$ and $\leqslant y$.)

Lemma 1: Let $\underline{z}, \bar{z} \in S$ be profiles such that $\underline{z} \leqslant \bar{z}$, let $\underline{B}_{n}(x)$ and $\bar{B}_{n}(x)$ denote the smallest and largest best responses for $n$ to any $x \in S$, and let $\underline{B}(x)$ and $\bar{B}(x)$ denote the collections $\underline{B}_{n}(x)$ and $\bar{B}_{n}(x), n \in N$. Then $\sup U([\underline{z}, \bar{z}])=\bar{B}(\bar{z})$ and $\inf U([\underline{z}, \bar{z}])=\underline{B}(\underline{z})$, and $\bar{U}([\underline{z}, \bar{z}])=[\underline{B}(\underline{z}), \bar{B}(\bar{z})]$.

Proof of Lemma: In view of Theorems 1 and 2, the largest and smallest best responses are well defined. By definition, $\underline{B}(\underline{z})$ and $\bar{B}(\bar{z})$ are in $U([\underline{z}, \bar{z}])$, and thus $[\underline{B}(\underline{z}), \bar{B}(\bar{z})] \subset \bar{U}([\underline{z}, \bar{z}])$. Suppose $z \notin[\underline{B}(\underline{z}), \bar{B}(\bar{z})]$ and, in particular, sup- 
pose $z_{n} \nless \hat{z}_{n} \equiv \bar{B}_{n}(\bar{z})$. We claim that $z_{n} \notin U[\underline{z}, \bar{z}]$ because $\hat{z}_{n} \wedge z_{n}$ strongly dominates $z_{n}$. Indeed, for any $x \in[\underline{z}, \bar{z}]$,

$$
\begin{aligned}
f_{n}\left(z_{n}, z_{-n}\right)-f_{n}\left(\hat{z}_{n} \wedge z_{n}, x_{-n}\right) & \leqslant f_{n}\left(z_{n}, \bar{z}_{-n}\right)-f_{n}\left(\hat{z}_{n} \wedge z_{n}, \bar{z}_{-n}\right) \\
& \leqslant f_{n}\left(\hat{z}_{n} \vee z_{n}, \bar{z}_{-n}\right)-f_{n}\left(\hat{z}_{n}, \bar{z}_{-n}\right) \\
& <0 \quad \text { by }
\end{aligned}
$$

where the last inequality uses the fact that $\hat{z}_{n} \vee z_{n}>\hat{z}_{n}$. Similarly, if $z_{n} \neq \underline{B}_{n}(\underline{z})$, then $z_{n}$ is strongly dominated by $z_{n} \vee \underline{B}_{n}(\underline{z})$. Then $\sup U([\underline{z}, \bar{z}])=\bar{B}(\bar{z})$ and $\inf U([\underline{z}, \bar{z}])=\underline{B}(\underline{z})$, and consequently $\bar{U}([\underline{\underline{z}}, \bar{z}])=[\underline{B}(\underline{z}, \bar{B}(\bar{z}))]$.

Proof of Theorem 5: Let $\bar{y}^{0}=\sup (S)$ and $y^{0}=\inf (S)$ and, for $k \geqslant 1$, define $\bar{y}^{k}=\bar{B}\left(\bar{y}^{k-1}\right)$ and $\underline{y}^{k}=\underline{B}\left(\underline{y}^{k-1}\right)$. We first show that $U^{k}(S) \subset\left[\underline{y}^{k}, \bar{y}^{k}\right]$. Indeed, this is true for $k=0$; suppose it is true for $k \leqslant j$. Then,

$$
U^{j+1}(S) \subset U\left(\left[\underline{y}^{j}, \bar{y}^{j}\right]\right) \subset\left[\underline{y}^{j+1}, \bar{y}^{j+1}\right],
$$

where the first containment follows from our earlier observation that $U$ is monotone nondecreasing in the set containment order, and the second follows from the lemma. These inclusions also establish that $\left\{\bar{y}^{k}\right\}$ is nonincreasing and $\left\{y^{k}\right\}$ is nondecreasing. Then, since the strategy lattice is complete, these séquences have order limits $\bar{y}=\inf \bar{y}^{k}$ and $y=\sup \underline{y}^{k}$. So, the serially undominated strategies all lie in the interval $[y, \bar{y}]$.

We now show that $y$ (and similarly $\bar{y}$ ) is itself serially undominated by showing that it is a Nash equilibrium profile. For if it is not, then there exists some $n$ and $x_{n}$ such that

$$
f_{n}\left(x_{n}, \underline{y}_{-n}\right)-f_{n}\left(\underline{y}_{n}, \underline{y}_{-n}\right)>0 \text {. }
$$

But then, by (A2),

$$
f_{n}\left(x_{n}, \underline{y}_{-n}^{k}\right)-f_{n}\left(\underline{y}_{n}^{k+1}, \underline{y}_{-n}^{k}\right)>0
$$

for some finite $k$, contradicting the best response property.

Q.E.D.

Theorem 5 has several important corollaries.

Corollary: Assume (A1)-(A4). Then there exists a pure Nash equilibrium. Moreover, there exist largest and smallest pure Nash equilibria in the given order.

Corollary: Assume (A1)-(A4). If the game $\Gamma$ has a unique pure Nash equilibrium, then $\Gamma$ is dominance solvable.

Corollary: Assume (A1)-(A4) and suppose, in addition, that the game $\Gamma$ is symmetric (unchanged by permutations of the player indexes). If $\Gamma$ has a unique pure symmetric equilibrium, then it is dominance solvable. 
Proof: Since the game is symmetric, all players have the same largest and smallest serially undominated strategies and the profiles of largest and smallest strategies are, by Theorem 5, pure symmetric Nash equilibria. If the game has but one pure symmetric Nash equilibrium, then the largest and smallest serially undominated strategies for any player must coincide. As we have seen the dominance solution is contained in the serially undominated set.

Q.E.D.

Lippman, Mamer, and McCardle (1987) have established a comparative statics theorem for an equilibrium selection from families of games characterized by increasing best response functions satisfying (A5) below. We obtain a related result, but one that identifies particular monotonic selections, namely, the maximum and minimum equilibria, and that does so for supermodular games, which may have multiple best responses. Sobel (1988) has independently provided a version of our result for games with Euclidean strategy spaces. For our analysis, let the payoff functions be parameterized by $\tau$ in some partially ordered set $T$, so that $f_{n}=f_{n}\left(x_{n}, x_{-n}, \tau\right)$. Assume:

(A5) $\quad f_{n}$ has increasing differences in $x_{n}$ and $\tau$ (for any fixed $x_{-n}$ ).

For smooth supermodular games, (A5) is implied by the following condition:

(A5') $\quad \partial^{2} f_{n} / \partial x_{n i} \partial \tau \geqslant 0$ for all $n$ and $i$.

THEOREM 6: Suppose that $\left\{N,\left(S_{n}, f_{n}\left(x_{n}, x_{-n}, \tau\right), n \in N\right), \geqslant\right\}$ is a family of supermodular games satisfying (A5). Then, the smallest and largest serially undominated strategies $\underline{x}_{n}(\tau)$ and $\bar{x}_{n}(\tau)$ are nondecreasing functions of $\tau$.

Proof: Let $\bar{B}(x, \tau)$ be the largest best response function as defined in the proof of Theorem 4 for the game with parameter $\tau$. By Topkis' Monotonicity Theorem, $\bar{B}_{n}\left(x_{-n}, \tau\right)$ is a nondecreasing function, so $\bar{B}(x, \tau)$ is nondecreasing. Every Nash equilibrium satisfies $\bar{B}(x, \tau) \geqslant x$. By Tarski's Theorem, $\bar{x}(\tau)=$ $\sup \{x \mid \bar{B}(x, \tau) \geqslant x\}$ is a fixed point of $\bar{B}(\cdot, \tau)$, so it is the largest Nash equilibrium. Since $\bar{B}(x, \cdot)$ is nondecreasing, $\bar{x}(\cdot)$ is nondecreasing. A similar argument applies to the smallest equilibrium.

Q.E.D.

Corollary: Assume (A1)-(A5). Then the largest and smallest pure Nash equilibria are nondecreasing functions of $\tau$.

Finally, we turn to the welfare theorem mentioned in the introduction.

THEOREM 7: Let $\underline{x}_{n}$ and $\bar{x}_{n}$ denote the smallest and largest elements of $S_{n}$ and suppose $y$ and $z$ are two equilibria with $y \geqslant z$. (1) If $f_{n}\left(\underline{x}_{n}, x_{-n}\right)$ is increasing in $x_{-n}$, then $f_{n}(y) \geqslant f_{n}(z)$. (2) If $f_{n}\left(\bar{x}_{n}, x_{-n}\right)$ is decreasing in $x_{-n}$, then $f_{n}(y) \leqslant f_{n}(z)$. If the condition in (1) holds for some subset of players $N_{1}$ and the condition in (2) holds for the remainder $N \backslash N_{1}$, then the largest equilibrium is the most preferred equilibrium for the players in $N_{1}$ and the least preferred for the remaining players, 
while smallest equilibrium is least preferred by the players in $N_{1}$ and most preferred by the remaining players.

Although the proof is obvious and the Theorem involves additional conditions not implied by the definition of supermodular games, the Theorem is useful because its conditions are satisfied in nearly all of the cited applications. In several of the games, $f_{n}\left(\underline{x}_{n}, x_{-n}\right)$ is increasing in $x_{-n}$ for all the players, and then the largest equilibrium $\bar{x}$ is Pareto-best and the smallest is Pareto-worst. If, in addition, the game is a smooth supermodular game and $\bar{x}_{n}$ is strictly less than the upper bound of the $n$ 's strategy set, then even the Pareto-best equilibrium is not a Pareto optimum. There are then positive externalities and there is a Pareto-preferred (but not equilibrium) strategy profile in which each player adopts a higher strategy. These observations are important for applied work, but they are also elementary to prove, so we do not pause to develop them here.

\section{ADAPTIVE DYNAMICS}

The problem of developing a convincing learning theory to test the "dynamic stability" of equilibrium in games has attracted considerable attention. Cournot (1838) and Edgeworth (1925), in their classic studies of oligopoly, employed the model that we now call "best response dynamics" (according to which each player in each round expects that his competitors will do the same thing they did at the last round, and optimizes accordingly). This model, however, is unsatisfying, partly because it is so arbitrary in its specificity about what people expect, and partly because it attributes extreme foolishness to the players. Indeed, the players' forecasts in this model can be regularly and predictably wrong, and can even lead to cyclic behavior that the players somehow fail to notice.

An alternative to the best response model, called "fictitious play," was introduced by Brown (1951). Under fictitious play, the players attribute to their competitors mixed strategies with probability weights that coincide with the empirical distribution of the past play. Initial results were promising: Robinson (1951) had proved that the method always converges to an equilibrium for two player, zero-sum, finite strategy games. However, this line of research atrophied after Shapley (1964) established that fictitious play can lead to an infinite pattern of cycling behavior for two player, finite strategy, general sum games.

Research in adaptive learning in games continues. Recently, Fudenberg and Kreps (1988) have investigated limiting behavior in a class of learning models for general extensive form games. The behavior they consider is broad enough to encompass Bayesian learning (regarded by some as the paradigm of rational learning). They conclude that learning may, even in the long-run, yield a larger set of strategies than is identified by Nash equilibrium.

Shapley and Fudenberg-Kreps establish the rather negative conclusion that Nash equilibrium play is not the only possible outcome of learning in general 
games. For supermodular games, however, sharper and more positive results are possible. To study this matter, let $\{x(t)\}, x(t) \in S, t \in T$, denote a "learning process." We are deliberately vague about whether the time index $t$ is discrete or continuous; our theory applies to both cases.

We require one bit of notation. Given a process $\{x(t)\}$, let $P(T, t)$ denote the strategies played between times $T$ and $t: P(T, t)=\{x(s) \mid T \leqslant s<t\}$.

$$
\begin{aligned}
& \text { A process }\{x(t)\} \text { is one of adaptive dynamics if }(\forall T)\left(\exists T^{\prime}\right)\left(\forall t \geqslant T^{\prime}\right) \\
& x(t) \in \bar{U}([\inf (P(T, t)), \sup (P(T, t))]) .
\end{aligned}
$$

Condition (A6) defines a very broad class because it imposes such a weak restriction on the players' choices. It requires only that, for any date $T$, there is a later date after which each player selects either a strategy that is "justifiable" in terms of the competitors' play since $T$ or, failing that, at least is in the interval defined by the set of such justifiable choices. Thus, for example, in continuous time with isolated serially undominated points, the player's choices might traverse a path between two of these, and still be justified. Here, "justify" is used in a very weak sense. A strategy choice is justified if there is no other strategy that would have done better against every combination of strategies in the smallest interval containing the competitors' recent past play. For example, in a three firm Bertrand game, if firms two and three have set price combinations $\left(p_{2}, p_{3}\right)$ of $(2,5)$ and $(3,4)$ in the past, then firm one can justify any choice which is a best response to some probability distribution over the interval of price pairs $[2,3] \times[4,5]=[(2,4),(3,5)]$. Nevertheless, we have the following theorem.

THEOREM 8: Let $\{x(t)\}$ be an adaptive dynamic process and let $\underline{x}=\inf (S)$ and $\bar{x}=\sup (S)$. Then for every supermodular game $\Gamma$,

$$
(\forall k)\left(\exists T_{k}\right)\left(\forall t \geqslant T_{k}\right) x(t) \in\left[\underline{B}^{k}(\underline{x}), \bar{B}^{k}(\bar{x})\right],
$$

where $\underline{B}^{k}(x)=\underline{B}\left(\underline{B}^{k-1}(x)\right)$, and similarly for $\bar{B}^{k}(x) .^{13}$

Proof: The conclusion holds trivially for $k=0$. Suppose it holds also for $k=j-1$. Note that $S$ is exactly the set of points in $S$ between $\bar{x}$ and $\underline{x}$, so

\footnotetext{
${ }^{13}$ Theorem 8 is stated for time paths $\{x(t)\}$ and may often be usefully applied to stochastic learning models by considering the paths they generate and subsequences of the path. For example, Fudenberg and Kreps (1988) study a class of models in which the players conduct an infinite number of experiments over time so that their behavior does not satisfy the requirements of (A6). Nevertheless, the stochastic behavior generated by their model does satisfy (A6) (with probability one) when $t$ is restricted to the subsequence of dates at which no experiments are conducted. The conclusion of Theorem 8 and its Corollaries then applies along that subsequence. In particular, the second corollary then implies that for finite strategy supermodular games with Fudenberg-Kreps learning, the play $x(t)$ almost surely lies eventually in the interval bounded by the smallest and largest Nash equilibria, except at those isolated dates when players are conducting experiments.

Applying a model like the Fudenberg and Kreps model to normal form games, we find that the resulting behavior satisfies (A6) along subsequences even in models in which the players do not know their competitors' payoffs or strategy sets or even how many other players there may be, but simply experiment by choosing strategies and pick the one that does best on average. For a fuller development of these ideas, including extensions to general games, see Milgrom and Roberts (1989).
} 
$S=[\underline{x}, \bar{x}]$. By the inductive hypothesis, for $t \geqslant T_{j-1}, x(t) \in\left[\underline{B}^{j-1}(\underline{x}), \bar{B}^{j-1}(\bar{x})\right]$. Applying (A6) with $T=T_{j-1}$ and letting $T_{j}=T^{\prime}$, we find that for all $t \geqslant T_{j}$,

$$
\begin{aligned}
x(t) & \in \bar{U}([\inf (P(T, t)), \sup (P(T, t))]) \subset \bar{U}\left\{\left[\underline{B}^{j-1}(\underline{x}), \bar{B}^{j-1}(\bar{x})\right]\right\} \\
& =\left[\underline{B}^{j}(\underline{x}), \bar{B}^{j}(\bar{x})\right],
\end{aligned}
$$

where the last step follows from Lemma 1.

Q.E.D.

COROLlaRY: Let $\{x(t)\}$ be an adaptive dynamic process for a supermodular game $\Gamma$ and let $y$ and $\bar{y}$ be the smallest and largest Nash equilibrium strategy profiles of $\Gamma$. Then, $\lim \inf x(t) \geqslant \underline{y}$ and $\lim \sup x(t) \leqslant \bar{y}$.

Corollary: For any adaptive process $\{x(t)\}$ and any finite strategy supermodular game, there exists a date after which $x(t)$ is bounded above and below by the largest and smallest Nash equilibrium strategy profiles.

\section{EXAMPLES OF SUPERMODULAR GAMES}

We offer five examples to illustrate various issues in applying the theory of supermodular games. The first, a Diamond-type search model, demonstrates the standard procedure for checking supermodularity in smooth, finite player games with real-valued strategies. The second, a Bertrand model, shows how ordinal transforms of the payoff function can be used to extend the range of application of the theorems. Example 3 illustrates the application of the theory to an infinite dimensional strategy space arising in the context of an arm's race. The fourth example uses the Hendricks-Kovenock model of oil drilling to illustrate the handling of uncertainty and private information. Our final example is based on the Milgrom-Roberts theory of modern manufacturing. It illustrates the use of supermodular games to study coordination failures among a group of people who share a common goal (a "team").

(1) A Diamond-type Search Model. There are a finite number of players $N$ who exert effort searching for trading partners. Any trader's probability of finding another particular trader is proportional to his own effort and the total effort of the others. Let $x(n) \in[0, \bar{x}]$ denote the effort of player $n$. Then, the payoff to player $n$ is defined by:

$$
f_{n}(x)=\alpha x(n) \sum_{m \neq n} x(m)-C(x(n)) .
$$

Since $\partial^{2} f_{n} / \partial x(n) \partial x(m)=\alpha>0$ (for $m \neq n$ ), this is a supermodular game. Suppose that $C(\cdot)$ is increasing. Then $x(n) \equiv 0$ is an equilibrium. If $C(\cdot)$ is also smooth and convex, then for each $\varepsilon$ satisfying $\alpha(N-1) \varepsilon=C^{\prime}(\varepsilon)$, there is also a symmetric equilibrium in which each searcher selects effort level $\varepsilon$. These equilibria are Pareto ranked, as our welfare theorem indicates. As our comparative statics theorem indicates, the upper bound on the equilibrium search effort rises with $\alpha$. 
(2) Bertrand Oligopoly with Differentiated Products. Some Bertrand oligopoly games can be analyzed as supermodular games. For example, Topkis (1979) has shown that if the goods are substitutes with linear demand and costs and if the players' strategies are prices constrained to lie in an interval $[0, \bar{p}]$, then the game is supermodular. ${ }^{14}$

The domain of the theory can be considerably expanded, however, by considering monotone transformations of the payoff function. An important example is the case in which each firm $n$ produces with constant unit costs $c_{n}$ and faces a demand function $D_{n}(p)$ with the properties that the goods are substitutes and that the elasticity of demand is a nonincreasing function of the other firms' prices. Mathematically, this second condition is equivalent to requiring that $\partial^{2} \log \left(D_{n}\right) / \partial p_{n} \partial p_{m} \geqslant 0$ for $n \neq m$. This form of demand encompasses the logit $\left(D_{n}=k_{n} / \Sigma_{j \in N} k_{j} \exp \left[\lambda\left(p_{j}-p_{n}\right)\right], \lambda<0\right)$, CES $\left(D_{n}=y p_{n}^{r-1} / \Sigma_{j \in N} p_{j}^{r}\right.$, where $r<0),{ }^{15}$ and transcendental $\operatorname{logarithmic}{ }^{16}\left(\log \left(D_{n}\right)=\alpha^{n}+\sum_{j \in N} \beta_{j}^{n} \log \left(p_{j}\right)+\right.$ $\sum_{j \in N} \sum_{i \in N} \gamma_{i j}^{n} \log \left(p_{i}\right) \log \left(p_{j}\right)$ where $\beta_{n}^{n}<0, \gamma_{n n}^{n}<0, \beta_{j}^{n} \geqslant 0$, and $\gamma_{n j}^{n} \geqslant 0$ for $j \neq n)$, as well as all the demand functions that Topkis studied. ${ }^{17}$ The firm's log-profits are:

$$
\log \left[\left(p_{n}-c_{n}\right) D_{n}(p)\right]=\log \left(p_{n}-c_{n}\right)+\log \left[D_{n}(p)\right] .
$$

For all demand functions in this class, if we take the strategies to be $x_{n}=p_{n} \in$ $\left[c_{n}, \bar{p}\right]$, then $\left(\mathrm{A} 1^{\prime}\right)-\left(\mathrm{A} 4^{\prime}\right)$ are satisfied and the (log-transformed) game is supermodular.

In fact, for the cases of linear demand, CES, logit, and translog with the additional parameter restrictions that $\sum_{j \in N} \beta_{j}^{n}<0$ and $\sum_{j \in N} \gamma_{n j}^{n}<0$, we can do even better, establishing the uniqueness of the pure strategy Nash equilibrium. We illustrate for the cases of logit and CES. Let player $n$ 's strategy be $x_{n}=p_{n}$ for the case of logit demand and $x_{n}=\log \left(p_{n}\right)$ for CES demand, where we restrict $p_{n} \in\left[c_{n}, \bar{p}\right]$. (An order-preserving transformation of the strategy spaces does not affect supermodularity, but in this case it simplifies the analysis of uniqueness.) With these assumptions, it is routine to verify that $\left(\mathrm{A} 1^{\prime}\right)-\left(\mathrm{A} 4^{\prime}\right)$ are satisfied, so the game is supermodular. Letting $f_{n}(x)=\log \left[\left(p_{n}-c_{n}\right) D_{n}(p)\right]$, one can verify for logit and CES demand that for all $x$ :

$$
-\partial^{2} f_{n} /\left(\partial x_{n}\right)^{2}>\sum_{j \neq n} \partial^{2} f_{n} / \partial x_{n} \partial x_{j} .
$$

This is a "dominant diagonal" condition and it implies that the transformed game has a unique pure strategy equilibrium. Indeed, if there are multiple equilibria, then since the game is supermodular there are two, $x$ and $\hat{x}$, with $\hat{x} \geqslant x$ (since there are largest and smallest equilibria). Let $n$ be the player for

\footnotetext{
${ }^{14}$ Vives (1989) extends this analysis to the case of convex costs. Also, Topkis had shown that the result extends to the case where the demand function satisfies the "increasing differences" condition; however the linear demand function is the only commonly studied one with that property.

${ }_{16}^{15}$ See Varian (1978).

${ }_{17}^{16}$ See Christensen, Jorgensen, and Lau (1973).

${ }^{17}$ For additional examples and a fine treatment of Bertrand equilibrium in spatial models, see Caplin and Nalebuff (1989).
} 
whom $\hat{x}_{j}-x_{j}$ is largest. Then,

$$
\frac{\partial f_{n}}{\partial x_{n}}(\hat{x})-\frac{\partial f_{n}}{\partial x_{n}}(x)=\int_{0}^{1}\left\{\sum_{j \in N}\left(\hat{x}_{j}-x_{j}\right) \cdot \frac{\partial^{2} f_{n}}{\partial x_{n} \partial x_{j}}[t \hat{x}+(1-t) x]\right\} d t .
$$

The assumption that $x$ and $\hat{x}$ are equilibria with $\hat{x}_{n}>x_{n}$ requires that $\partial f_{n} / \partial x_{n}$ be nonnegative at $\hat{x}$ and nonpositive at $x$, so the left-hand side of (7) must be positive. However, according to (6), the integrand in (7) must be strictly negative, a contradiction. So, the equilibrium is unique.

Since the transformed game is supermodular and has a unique equilibrium, it follows from Theorem 5 that each player has only one serially undominated strategy. Since the set of serially undominated strategies is determined only by ordinal comparisons, the corresponding prices are the unique serially undominated strategies in the original game, as well. Hence, the original Bertrand game has a unique equilibrium and is actually dominance solvable, and the equilibrium is globally stable under any adaptive learning rule, that is, any adaptive rule satisfying (A6). Comparative statics are also transferrable between the two games. For example, an increase in any one cost $c_{n}$ results in an increase in all the firms' prices (by Theorem 6) in both the transformed and original games.

(3) Arms Races. The players are two countries engaged in an arms race. In the static version of the game, each player chooses a level of arms $x_{n} \in\left[0, x_{\text {Max }}\right]$ and receives as its payoff $f_{n}\left(x_{n}, x_{-n}\right)=-C\left(x_{n}\right)+B\left(x_{n}-x_{-n}\right)$, where $B$ is a smooth concave function and $C$ is a smooth function of any shape. The game is assumed to be symmetric, so the same payoff function applies to players 1 and 2 . Since $\partial^{2} f_{1} / \partial x_{1} \partial x_{2}=-B^{\prime \prime}\left(x_{1}-x_{2}\right) \geqslant 0$, this is a supermodular game.

One can extend this one-shot game to a dynamic game in which stocks of armaments accumulate and depreciate over time. We use "open loop" strategies $x_{n}$, where $x_{n}(t)$-the level of arms at time $t$-proceeds according to the dynamic equation $x_{n}(t)=(1-\delta) x_{n}(t-1)+I_{n}(t)$. Here $\delta$ is the rate of depreciation, $I_{n}(t)$ is the rate of investment in armaments at time $t$ (implied by the strategy $\left.x_{n}\right)$, and the initial condition is $x_{n}(-1)=0$. Suppose that the payoffs in the game are the present value of the advantages of superior armaments minus the cost of investment in armaments $C\left(I_{n}(t)\right)$, as follows:

$$
\sum_{t=0}^{\infty} \rho^{t}\left[B\left(x_{n}(t)-x_{-n}(t)\right)-C\left(x_{n}(t)-(1-\delta) x_{n}(t-1)\right)\right] .
$$

We now assume that $B$ is increasing as well as concave, that $C$ is increasing and convex, that $\rho$ and $\delta$ lie strictly between zero and one, and that there is some $\bar{I}$ such that $x_{n}$ is feasible if and only if $I_{n}(t)$ lies in $[0, \bar{I}]$ for all $t$. Let $x_{n} \geqslant \hat{x}_{n}$ mean that $x_{n}(t) \geqslant \hat{x}_{n}(t)$ for all $t$. Each term in the sum (8) satisfies the supermodularity, increasing differences, and continuity conditions (A2)-(A4). Veinott (1989) has shown that the properties of supermodularity and increasing differences are preserved under summation and that the pointwise limit of a sequence of such functions also satisfies these conditions. 
We verify (A1) as follows. First, we may regard the strategy sets as subsets of the lattice $\{x \mid 0 \leqslant x(t) \leqslant t \bar{I}\}$. The constraints on the strategy choices are $x_{n}(0)=0$ and $\bar{I} \geqslant x_{n}(t)-(1-\delta) x_{n}(t-1) \geqslant 0$, for $t=1,2, \ldots$. One can directly verify that each of these constraints individually describes a complete sublattice. Furthermore, it follows immediately from the definitions that any intersection of complete sublattices is a complete sublattice (and hence a complete lattice in its own right). So, all the conditions are satisfied and the game is supermodular.

The first order conditions for optimal investment at a symmetric equilibrium require $C^{\prime}\left(I_{n}^{*}(t)\right)=B^{\prime}(0) /[1-\rho(1-\delta)]$ for all $t$, from which the symmetric equilibrium strategy $x^{*}$ can be uniquely inferred. Consequently, the pure symmetric equilibrium is unique and the game, despite its infinite dimensional strategy space, is dominance solvable. At each stage $s$ in the dominance elimination process, there is an interval $\left[\underline{x}^{s}, \bar{x}^{s}\right]$ containing $x^{*}$ such that all remaining strategies $x$ must satisfy $\underline{x}^{s}(t) \leqslant x(t) \leqslant \bar{x}^{s}(t)$ or be strongly dominated at that stage by the strategy $\left(x \vee \underline{x}^{s}\right) \wedge \bar{x}^{s}$, which is obtained from $x$ by truncating it to lie within the prescribed interval. As $s$ increases, the strategies $\bar{x}^{s}$ converge monotonically down to $x^{*}$ while the strategies $\underline{x}^{s}$ converge monotonically up to $x^{*}$, as the proof of Theorem 5 demonstrates.

The corresponding continuous time arms race game can also be shown to be supermodular and dominance solvable. ${ }^{18}$

(4) Drilling for Oil. This game is based on a model of Hendricks and Kovenock (1989). The players are two oil firms who own similar tracts of land which may or may not bear oil. Each firm observes a signal $s_{n}$ (a geological report) at date 0 that provides information about the unknown, common value $v$ of the tracts; the signal is modeled as a real-valued random variable. Drilling costs are $c$ and the discount factor is $\delta$. If a firm drills at date $t(t=0,1)$, its payoff is $\delta^{t}(v-c)$. If one firm drills at date zero, then the other firm can condition its drilling decision on the outcome. In that case, if it delays drilling, its date 1 payoff will be $\max (0, v-c)$.

We consider a simplified version of the Hendricks-Kovenock game in which the game ends immediately with payoffs of zero if neither firm drills at date 0 . Let $\chi_{n}$ denote the indicator function of the set of signals that trigger drilling by firm $n$ at date 0 . These indicator functions describe the pure strategies available to each firm. We order the two players' strategy sets oppositely. Thus, we say that $\chi_{n} \leqslant \hat{\chi}_{n}$ if $(-1)^{n} \chi_{n}(t) \leqslant(-1)^{n} \hat{\chi}_{n}(t)$ for almost all $t$.

Suppose that the underlying value $v$ has finite expectation and that the joint density of the value and signals $\left(v, s_{1}, s_{2}\right)$ is $g\left(v, s_{1}, s_{2}\right)$. Payoffs for firm 1 are then as follows:

$$
\begin{aligned}
& \iiint(v-c) \chi_{1}(s) g(v, s, t) d v d s d t \\
& \quad+\delta \iiint \max (0, v-c) \chi_{2}(t)\left[1-\chi_{1}(s)\right] g(v, s, t) d v d s d t .
\end{aligned}
$$

\footnotetext{
${ }^{18}$ The one extra difficulty in the continuous time game is to establish that the payoff function is order upper semi-continuous, but this can be done with no extra assumptions.
} 
The first term is the firm's payoff from immediate drilling; the second is its payoff from not drilling in period zero when the second firm drills.

Notice that for each fixed value of $(v, t, s)$, the integrand is a supermodular function of $-\chi_{1}(s)$ and $\chi_{2}(t)$. As Vives (1989) first observed about Bayesian games of this sort, it follows that the payoff function is supermodular in the strategies with the given partial ordering. Since the payoff function is plainly $L_{1}$-continuous, Theorem 3 implies that the payoff functions are order-continuous and that the strategy sets are complete lattices. By our assumption that $E[v]$ is finite, the payoffs are bounded. So this is a supermodular game. As Hendricks and Kovenock have shown, such games can have multiple Nash equilibria. Since firm 1's payoff increases in $\chi_{2}$ and 2's payoff declines in $\chi_{1}$ (given our ordering), Theorem 7 implies that the best equilibrium for firm 1 and the worst for firm 2 is the one at which firm 2 drills most often.

(5) Modern Manufacturing. Our interest in supermodular games began with our research into modern manufacturing (Milgrom and Roberts (1990)), for which we constructed a model of the firm's technological, organizational, product design, and pricing decisions. In that model, we showed that the firm's payoff function is supermodular.

We now extend our previous analysis to study the coordination problem of a "team" of managers who run the firm and adapt to changing technological opportunities. That the managers form a team means that they all share the same payoff function $f$, which is also the payoff function of the firm. Thus, let $f\left(\theta^{1}, \ldots, \theta^{k}, \tau\right)$ be a supermodular function representing both the profits of the firm and the payoff of each manager when manager $j$ selects $\theta^{j}$ from a compact interval $I_{j} \subset \Re^{m_{j}}$ and when the exogenous parameter value is $\tau$. It is routine to check that the game played among the managers is a supermodular game. Consequently, the Corollary of Theorem 9 (about adaptive dynamics) applies to it. Clearly, the firm's optimum is a Nash equilibrium of the team game. If the game has a unique Nash equilibrium, then any behavior by the managers consistent with adaptive dynamics will lead the managers' behavior to converge to the optimum without any explicit coordination among them. If there are multiple equilibria, however, then there can be a coordination failure in which the managers' adaptive behavior fails to converge toward the firm's optimum.

One can further enrich the model by incorporating competition from other firms producing substitute goods. For example, suppose that the firm faces a linear demand function $Q_{n}=D\left(p_{n}, p_{-n}\right)$, that $x_{n}=\left(p_{n}, \theta_{n}^{1}, \ldots, \theta_{n}^{k}\right)$, and that $f_{n}(x, \tau)=p_{n} Q_{n}-C\left(Q_{n}, \theta_{n}, \tau\right)$, where marginal cost is nondecreasing and $-C\left(-Q_{n}, \theta_{n}, \tau\right)$ is supermodular. Suppose that each firm is a team of $k+1$ managers, of whom one sets the price and the other $k$ choose the $\theta_{n}^{j}$ 's. If there are $N$ firms, then this $N(k+1)$-player game is supermodular, and our comparative statics and adaptive learning results (Theorems 6 and 9) apply directly. In our earlier paper, we argued that the firm's optimum $\left(p^{*}(\tau), \theta^{*}(\tau)\right)$ was nondecreasing in $\tau$ if $f$ has increasing differences in $(p, \theta)$ and $\tau$. We now see that the same conclusion applies to the bounds on equilibrium behavior for an 
extended model with competing oligopolistic firms, each run by a team of managers using adaptive learning rules.

\section{CONCLUDING REMARKS}

Most attempts to analyze noncooperative games have focused on developing general solution concepts that could be applied to all such games. This approach seems not always to be attractive; there is no single story that applies equally to all game situations. Some games are played without precedent and among strangers; players then must reason about how their competitors will play and so how they themselves should play from whatever they know about the rules of the game and from their general knowledge about human behavior and the backgrounds of the other players. Under these circumstances, there is little reason to suppose that the players will have correct expectations about how their competitors will play, and then rationalizable strategies and correlated equilibrium provide attractive alternatives to the Nash equilibrium solution concept. In other situations, the institution being modeled is a stable one that has attracted new participants regularly in the past so that the behavior of past participants may be a good guide to that of the new ones. If the environment were truly stationary, it would be natural to use adaptive learning models to predict the kinds of behavior that might emerge. Most often, however, games do have some precedents, but not perfect ones, so that some combination of reasoning and observation will be what actually guides the players' choices. The class of supermodular games is remarkable and useful because it is a class that includes so many games that have been of greatest interest in applied theory and for which all these alternative "solutions" imply identical bounds on the joint behavior of the players.

Department of Economics and Graduate School of Business, Stanford University, Stanford, CA 94305, U.S.A.

Manuscript received January, 1989; final revision received March, 1990.

\section{REFERENCES}

Aliprantis, Charalambos, and Owen Burkinshaw (1985): Positive Operators. New York: Academic Press.

AumanN, Robert (1987): "Correlated Equilibrium as an Expression of Bayesian Rationality," Econometrica, 55, 1-18.

Bernheim, B. Douglas (1984): "Rationalizable Strategic Behavior," Econometrica, 52, 1007-1028.

BIRKhoff, G. (1967): Lattice Theory. Providence RI: American Mathematical Society.

Brown, G. W. (1951): "Iterative Solution of Games by Fictitious Play," Activity Analysis of Production and Allocation. New York: John Wiley and Sons.

BRYANT, John (1983): “A Simple Rational Expectations Keynes-Type Model," Quarterly Journal of Economics, 98, 525-528.

(1987): "The Paradox of Thrift, Liquidity Preference, and Animal Spirits," Econometrica, 55, $1231-1235$. 
Bulow, J. I., J. D. Geanakoplos, and P. D. Klemperer (1985): "Multimarket Oligopoly: Strategic Substitutes and Complements," Journal of Political Economy, 93, 488-511.

Caplin, Andrew, and Barry Nalebuff (1989): "Aggregation and Imperfect Competition: On the Existence of Equilibrium," mimeo, Columbia University, 1989. To appear in Econometrica.

Christensen, Lars, Dale Jorgensen, and Larry Lau (1973): "Transcendental Logarithmic Production Frontiers," Review of Economics and Statistics, 55, 28-45.

COOPER, Russell, AND ANDREW JoHN (1988): "Coordinating Coordination Failures in Keynesian Models," Quarterly Journal of Economics, 103, 441-463.

Cournot, Augustine (1838): Recherches sur les Principes Mathématiques de la Théorie des Richesses, 1838, translated by N. T. Bacon as Researches into the Mathematical Principles of the Theory of Wealth. London: Hafner, 1960.

Diamond, Douglas, and Philip Dybvig (1983): "Bank Runs, Deposit Insurance, and Liquidity," Journal of Political Economy, 91, 401-419.

Diamond, Peter (1982): “Aggregate Demand Management in Search Equilibrium," Journal of Political Economy, 90, 881-894.

Dybvig, Philip, and Chester Spatt (1983): “Adoption Externalities as Public Goods," Journal of Public Economics, 20, 231-247.

Edgeworth, F. (1925): "The Pure Theory of Monopoly," in Papers Relating to Political Economy, Vol. 1, ed. by F. Edgeworth. London: Macmillian.

Farrell, Joseph, and Garth Saloner (1986): "Installed Base and Compatibility: Innovation, Product Preannouncements, and Predation," American Economic Review, 76, 940-955.

FudENBERG, D., AND D. KREPS (1988): “A Theory of Learning, Experimentation, and Equilibrium in Games," unpublished manuscript.

Fudenberg, D., AND J. Tirole (1986): Dynamic Models of Oligopoly. Chur, Switzerland: Hardwood Academic Publishers.

$\rightarrow$ Granot, Frieda, and Arthur F. Veinott (1985): "Substitutes, Complements and Ripples in Network Flows," Mathematics of Operations Research, 10, 471-497.

Gul, FaruK (1988): "Strategic Behavior and the Notion of Equilibrium," mimeo, Stanford University.

Hendricks, Ken, ANd Dan Kovenock (1989): “Asymmetric Information, Information Externalities, and Efficiency: The Case of Oil Exploration," Rand Journal of Economics, 20, 164-182.

HiRsCH, MoRris (1982): "Systems of Differential Equations which are Competitive or Cooperative. I: Limit Sets," Siam Journal of Mathematical Analysis, 13, 167-179.

- (1985): "Systems of Differential Equations which are Competitive or Cooperative. II: Convergence Almost Everywhere," Siam Journal of Mathematical Analysis, 16, 423-439.

Katz, Michael, and Carl Shapiro (1986): "Technology Adoption in the Presence of Network Externalities," Journal of Political Economy, 94, 822-841.

Lippman, Steven, John Mamer, and Kevin McCardle (1987): "Comparative Statics in NonCooperative Games via Transfinitely Repeated Play," Journal of Economic Theory, 41, 288-303.

Milgrom, Paul, and John Roberts (1990): "The Economics of Modern Manufacturing: Technology, Strategy and Organization," American Economic Review, 80, 511-528.

(1989): "Adaptive and Sophisticated Learning in Repeated Normal Form Games," mimeo, Stanford University. To appear in Games and Economic Behavior.

Moulin, Herve (1984): "Dominance Solvability and Cournot Stability," Mathematical Social Sciences, 83-102.

Pearce, David (1984): "Rationalizable Strategic Behavior and the Problem of Perfection," Econometrica, 52, 1029-1050.

Reinganum, Jennifer (1981): "Dynamic Games of Innovation," Journal of Economic Theory, 25, 21-41.

Robinson, J. (1951): “An Iterative Method of Solving a Game," Annals of Mathematics, 54, 296-301.

Scitovsky, Tibor (1954): “Two Concepts of External Economies," Journal of Political Economy, 62, $70-82$.

Shapley, Lloyd (1964): "Some Topics in Two-Person Games," in Advances in Game Theory, Annals of Mathematical Studies, 5, 1-28.

Shannon, Christine (1989): Private communication, Stanford University.

SHAVELl, SteVEN (1989): "Sharing of Information Prior to Settlement or Litigation," Rand Journal of Economics, 20, 183-195. 
Sobel, Matrhew (1988): "Isotone Comparative Statics in Supermodular Games," mimeo, State University of New York, Stony Brook.

Spatt, Chester, and Frederic Sterbenz (1988): “Warrant Exercise, Dividends, and Reinvestment Policy," Journal of Finance, 42, 493-506.

TARSKI, A. (1955): "A Lattice-Theoretical Fixpoint Theorem and Its Applications," Pacific Journal of Mathematics, 5, 285-309.

Topkis, Donald M. (1979): "Equilibrium Points in Nonzero-Sum n-Person Submodular Games," Siam Journal of Control and Optimization, 17, 773-787.

(1978): "Minimizing a Submodular Function on a Lattice," Operations Research, 26, 305-321.

VARIAN, Hal (1978): Microeconomic Analysis. New York: W. W. Norton.

Veinott, ARthur F. (1989): "Lattice Programming," unpublished notes from lectures delivered at Johns Hopkins University.

VIVES, XAVIER (1985): "Nash Equilibrium in Oligopoly Games with Monotone Best Responses," CARESS Working Paper No. 85-10, University of Pennsylvania.

(1989): "Nash Equilibrium with Strategic Complementarities," mimeo, Universitat Autonoma de Barcelona. To appear in the Journal of Mathematical Economics. 